\title{
Enhancement of Superthermal Ultracold Neutron Production by Trapping Cold Neutrons*
}

\author{
P. D. Bangert, ${ }^{* *}$ M. D. Cooper, and S. K. Lamoreaux \\ Los Alamos National Laboratory \\ Los Alamos, NM 87545, USA.
}

\begin{abstract}
In the production of ultracold neutrons (UCN) by scattering cold neutrons in superfluid ${ }^{4} \mathrm{He}$ or cold solid $\mathrm{D}_{2}$ (“superthermal" processes), the UCN production rate is proportional to the spatial density of cold neutrons in the material. For a superthermal source fed from a cold neutron guide, we show that the rate of UCN production can be increased by up to a factor of 2.5 for realistic substances by enclosing the source in a cavity made of a material with a high cold neutron albedo.
\end{abstract}

\footnotetext{
* Work supported in part by the U.S. DOE.

** Current address: Department of Physics and Astronomy, University College London, Gower St., London WC1E 6BT, UK.
} 


\section{Introduction}

The most promising avenue for increasing the density of ultracold neutrons (UCN) available for experiments is in the use of "superthermal" processes [1]; a relatively high energy neutron (corresponding to a temperature of $10-40^{\circ} \mathrm{K}$ ) can produce a phonon in a cold material such as superfluid ${ }^{4} \mathrm{He}$, and come to near rest (temperature less than $5 \mathrm{mK}$ ) [2]. The inverse process, that is, a UCN absorbing a phonon from the material and having its energy increased above the UCN range, is suppressed by the Boltzmann factor. The most famous example of a superthermal source is based on superfluid ${ }^{4} \mathrm{He}[1]$. If the superfluid bath is below $0.7^{\circ} \mathrm{K}$, the mean $\mathrm{UCN}$ lifetime against upscattering is equal to the beta decay lifetime (neutron absorption by ${ }^{4} \mathrm{He}$ is energetically forbidden), while the production rate is essentially independent of temperature:

$$
P=\left.7.2 \frac{d \Phi}{d \lambda}\right|_{8.9 \AA} \frac{1}{\lambda^{3}},
$$

where the flux is specified as the number of neutrons incident on the source per second

$\mathrm{cm}^{2} \AA$, and $\lambda_{u}$ corresponds to the shortest wavelength UCN that can be contained by the walls surrounding the superthermal source. The UCN density is then given by the UCN lifetime $\tau$ in the container, times the production rate $P$ given above. For modern UCN storage techniques, $\tau$ can approach the neutron beta decay lifetime of $\sim 900 \mathrm{~s}$; in this case, at existing cold neutron sources, UCN densities of up to $10^{3}$ times existing sources might be possible.

The use of superfluid ${ }^{4} \mathrm{He}$ as a UCN source is best applied to a specific experiment, such as has been proposed for the neutron electric dipole moment (EDM) [3] or neutron 
lifetime [4], where the UCN are available, albeit at high density, only after a rather long accumulation time. Another type of superthermal source is based on solid $\mathrm{D}_{2}$ or other cold material; this type of source is best thought of as a "current source" with a high density [5] of UCN continuously available.

In order to maximize the UCN production rate from a superthermal source, it should be placed as close as possible to the source of cold incident neutrons. This arrangement is possible because the UCN production rate does not depend on collimation of the incident neutrons, but only on the neutron density. However, for a number of practical reasons, it is easier to feed a superthermal source from a neutron guide because placing a very cold material near a reactor or spallation cold source would require considerable cryogenic resources. For a number of applications (testing UCN production in solid $\mathrm{D}_{2}$ [3], a neutron EDM experiment [2], a neutron beta-decay lifetime measurement [4]), feeding the superthermal source from a neutron guide is a quite viable alternative; in fact, with modern supermirror guide technology, the effective solid angle subtended by the guide can approach the maximum solid angle (due to restrictions on radiation heating) that one could hope to achieve by placing the superthermal source near the cold source.

As we describe below, it is possible to enhance the UCN production rate for a superthermal source located on a neutron guide. Neutrons exiting a guide are restricted within a very narrow solid angle. The incident neutrons can therefore be trapped in a cavity made of a high, diffuse reflectivity (albedo) material, and their interaction time or effective density can be increased, in principle, by up to a factor of four. This improvement is due in part to the fact that materials useful for a superthermal UCN source have very low neutron 
absorption rates; in the case of solid $\mathrm{D}_{2}$ or $\mathrm{CD}_{4}$, the absorption lifetimes are of order 0.15 sec.

\section{Kinetic Theory of a Cold Neutron Trap}

Consider the apparatus shown in Fig. 1. Cold neutrons leave a guide and enter a cavity of internal volume $V$ and internal area $A$ through an aperture of area $a$. If the cavity wall has zero albedo, the effective neutron density in the cavity is given by

$$
\rho_{c}^{0}=\rho_{b} a d / V,
$$

where $\rho_{c, b}$ are the beam and cavity neutron densities, and $d$ is the characteristic length indicated in Fig. 1. The beam density is simply given by

$$
\rho_{b}(v)=\Phi(v) / v,
$$

where $\Phi(v)$ is the incident flux at velocity $v$. In the case of a superfluid ${ }^{4} \mathrm{He}$ superthermal source, we are only interested in a narrow velocity range; in the discussion below, we will consider a single fixed neutron velocity component.

Now consider the case where the cavity walls have a finite average reflectivity $R$. $\rho_{c}$ can be estimated by balancing the rate at which neutrons enter the cavity (per unit volume) to the rate at which they exit (either through losses at the wall reflections or through the entrance aperture); 


$$
\rho_{c}=\rho_{c}^{0}+R \frac{\Phi a}{V} \tau
$$

where $\rho_{c}^{0}$ is the density when $R=0$. The factor of $R$ in the second term gives the probability that the neutrons are trapped and gives the input rate for the following statistical calculation.

The total lifetime for a cold neutron in the cavity is given by

$$
\tau^{-1}=\tau_{a}^{-1}+\tau_{w}^{-1}
$$

where the wall loss rate is given by $\tau_{w}^{-1}=(1-R)(v / \bar{L})$ with

$$
\bar{L}=\frac{4 V}{A}
$$

the mean free path from kinetic theory [6]. The loss rate through the aperture is determined by the flux incident on it,

$$
j=\frac{1}{4} \rho_{c} v
$$

giving a loss time constant for $N=\rho_{c} V$ neutrons in the cavity of

$$
\tau_{a}^{-1}=\frac{\dot{N}}{N}=\frac{\frac{1}{4} \rho_{c} v a}{\rho_{c} V}=\frac{1}{4} \frac{v a}{V} .
$$


Algebraically combining the above equations gives the relative density in the cavity compared to the incident beam,

$$
\frac{\rho_{c}}{\rho_{b}}=\frac{a L}{V}+\frac{4 R}{(A / a)(1-R)+1},
$$

which can be as large as 4 in the limit of $R$ tending to 1 . The actual enhancement factor is the density in the cavity with the reflector as compared to the density without the reflector

$$
\frac{\rho_{c}}{\rho_{c}^{0}}=1+\frac{4 V R}{d A(1-R)+a d} .
$$

Insofar as we have taken a single velocity class of neutrons as appropriate for superthermal sources, the cavity enhancement $\rho_{c} / \rho_{c}^{0}$ does not depend on the neutron velocity (though, in general, $R$ is a function of $v$ ).

In the limit that $R \rightarrow 1$ we see an enhancement in the cavity density by a factor of over four. The implication is that the cavity, if constructed from high enough albedo material, will compensate for a guide of cross sectional area $a<<A$, and we can enjoy an embellished cold neutron density over a much larger bottle cross section than implied by the guide area. 


\section{Fermi's Albedo Principle}

In actual experiments, the statistical precision of the result depends on the total number of UCN in the sample per unit time. The figure of merit for a reflector design is the probability that a neutron scatters in the source multiplied by the volume of the source vessel. The probability of interaction is proportional to the distance traveled in the vessel, and we can obtain the enhancement due to the reflector just by comparing the distances traversed with and without the reflector.

We construct an elementary model of the source by considering the average reflectivity of the walls $\bar{R}$, the cross-sectional area of the guide $a$, and the surface area of the reflector $A$. The probability of a neutron being reflected from the wall $\varepsilon$ is

$$
\varepsilon=\bar{R}(1-a / A) .
$$

The average number of intersections with a wall is denoted by $N$. We have

$$
N=\sum_{n=0}^{\infty} \varepsilon^{n}=\sum_{n=0}^{\infty}\left[\bar{R}^{n}\left(1-\frac{a}{A}\right)^{n}\right]=\frac{A}{A-\bar{R}(A-a)} .
$$

The total distance traveled in the sample per neutron is

$$
L=d+N \bar{L},
$$

where $\bar{L}$ is the mean free path in the sample and $d$ is the distance that the neutrons travel from the guide to their first intersection with the wall (see Fig. 1). From statistical 
mechanics, it can be shown that $\bar{L}=(\alpha V / A)$, where $V$ is the volume of the cavity and $\alpha$ is approximately 4 (see Ref. [6], p. 27). This expression is roughly independent of the geometry of the cavity. One can realistically expect the approximation $\alpha=4$ to be accurate to within 20 percent. We thus find

$$
L=d+4 V\left[\frac{1}{A-\bar{R}(A-a)}\right] .
$$

The reflectivity of the cavity walls $R$, as a function of incident angle $\tau$, can also be calculated theoretically as was done by Fermi (see Ref. [7], p. 496) for an infinite plane of reflector material. The material is characterized by the number of scattering collisions per absorbing collision $n$, given by the ratio of the elastic incoherent scattering cross section $\sigma_{\mathrm{inc}}$ to the absorption cross section $\sigma_{\mathrm{abs}}$.

$$
n=\frac{\sigma_{\text {inc }}}{\sigma_{\mathrm{abs}}}, \quad R=\frac{\sqrt{n}-1}{\sqrt{n}+\sqrt{3} \cos \theta} .
$$

From $R$, the average reflectivity $\bar{R}$ is computed by noting that the incident angles are distributed according to $\cos (\theta)$.

$$
\bar{R}=\beta \int_{0}^{\pi} \cos \theta R d \theta=\beta(\sqrt{n-1})\left[\frac{\pi}{2 \sqrt{3}}-2 \sqrt{\frac{n}{3 n-9}} \tan ^{-1}\left(\frac{\sqrt{n-3}}{\sqrt{n}+\sqrt{3}}\right)\right] .
$$

Since Fermi's model is for an infinite plane of infinite thickness, we introduce a factor $\beta$ that takes the finite geometry into account. Generally $\beta$ will be a function of 
thickness, size of the vessel, and material. The enhancement factor that the reflector gives the experiment is $E_{t}=L d$. Collecting the above expressions together, we have

$$
E_{t}=1+\frac{4 V}{d}\left[\frac{1}{A-(A-a) \beta(\sqrt{n}-1)\left[\frac{\pi}{2 \sqrt{3}}-2 \sqrt{\frac{n}{3 n-9}} \tan ^{-1}\left(\frac{\sqrt{n-3}}{\sqrt{n}+\sqrt{3}}\right)\right]}\right]
$$

The number of neutrons in the cavity at any time can be found in terms of the flux of neutrons per unit area in the guide $\Phi_{0}$ and the neutron velocity $v$,

$$
M_{t}=\frac{\Phi_{0} a d E_{t}}{v}
$$

\section{Monte Carlo Analysis}

To test the simple theory, we have performed a Monte Carlo analysis of some reflectors. We have tried to optimize the design to maximize the enhancement factor and the total number of neutrons in the ${ }^{4} \mathrm{He}$, subject to some engineering constraints imposed by the design of an EDM experiment, an interest of ours. The neutrons considered in the following sections are monoenergetic with a wavelength of $8.9 \AA$.

A computer simulation of the neutron transport through the apparatus was constructed for cylindrical (see Fig. 1) and rectangular geometries. The neutrons were uniformly distributed throughout the guide and collided with the wall on the opposite side. Then they were transported through the apparatus according to the following scheme: 
1. Choose a diffusion length $l$ according to the exponential distribution $f(l)=$ $\left(1 / l_{0}\right) e^{-l l l_{0}}$, where $l_{0}=\left[1 /\left(\sigma_{\mathrm{inc}}+\sigma_{\mathrm{abs}}\right) \rho\right]$ and $\rho$ is the density of atoms in the material.

2. Move the neutron by this length.

3. Determine if the neutron was absorbed in this collision according to the fractional probability of absorption $\sigma_{\mathrm{abs}} /\left(\sigma_{\mathrm{inc}}+\sigma_{\mathrm{abs}}\right)$.

4. If there is no absorption, scatter the neutron uniformly into a solid angle of $4 \pi$.

5. If the neutron emerges from the material, determine if it is transmitted. If it is inside the source, propagate it to the boundary. At this point it is either lost back into the guide or the process commences again.

This procedure is carried out for many neutrons and the Monte-Carlo enhancement factor $E_{\mathrm{MC}}$ is computed as the average ratio of the total distance that a neutron travels through the ${ }^{4} \mathrm{He}$ to the length of the cell, i.e., the distance that the neutron would have traveled through the ${ }^{4} \mathrm{He}$ if there was no reflector. We also compute the number of neutrons $M_{\mathrm{MC}}$ from the enhancement factor $E_{\mathrm{MC}}$ and the dimensions of the apparatus via Eq. (18). The simulated values $E_{\mathrm{MC}}$ and $M_{\mathrm{MC}}$ are compared to the theoretical ones. They agreement is expected to be about 40 percent due to the various idealizations assumed in the theory.

Vital inputs to simulating a particular material are the density and the cross sections for incoherent scattering and absorption. We have calculated the cross sections for compounds from the data for particular elements [8]. For $N$ constituent atoms of a molecule, we combine the bound coherent scattering lengths for each component atom, $a_{i}$, to form the total and coherent scattering cross sections, $\sigma_{\text {tot }}$ and $\sigma_{\text {coh }}$, respectively, via 


$$
\sigma_{\mathrm{coh}}=4 \pi N\left(\sum_{i=1}^{n} f_{i} a_{i}\right)^{2}, \quad \sigma_{\mathrm{tot}}=4 \pi \sum_{i=1}^{n} N_{i} a_{i}^{2}
$$

where $f_{i}=N_{i} / N$ is the number fraction of the $\mathrm{i}^{\text {th }}$ atom. The incoherent scattering cross section is the difference between the two.

$$
\sigma_{\text {inc }}=\sigma_{\text {coh }}-\sigma_{\text {tot }}
$$

If each constituent atom has an absorption cross section of $\sigma_{i}^{\mathrm{abs}}$, we take the total absorption cross section of the compound at an absolute temperature $T$ to be [8]

$$
\sigma_{\mathrm{abs}}=\sqrt{\frac{300}{T}} \sum_{i=1}^{n} \sigma_{i}^{\mathrm{abs}}
$$

where it is assumed that the values in the tables are given at the standard temperature of $300^{\circ} \mathrm{K}$. Table 1 lists the cross sections and densities for the materials used in the following analysis. The cross sections for $\mathrm{BeO}$ are unreliable because they depend on the method of preparation. The potential grainy structure of the material may raise the cross section from the value of 1 barn given by Eq. (20) to the value we used of 20 barns.

We determined the thickness of a material that essentially saturates the enhancement factor. We have computed $E_{\mathrm{MC}}$ for several materials and thicknesses. The results, for a rectangular geometry with the height taken as $10 \mathrm{~cm}$ and with $d$ set to $20 \mathrm{~cm}$, are shown in Table 2. 
Table 2 also lists the $\beta$ factors obtained for each material and the theoretical enhancement factor $E_{t}$ that results from the $\beta$ factor and the material properties. The theoretical enhancement is compared to the enhancement factor at the corresponding saturation thickness. We note that the percentage differences between $E_{t}$ and $E_{\mathrm{MC}}$ are well within the expected 40 percent accuracy of the theory.

In the relative evaluation of materials, the practical aspects of a particular apparatus must be taken into account. Since the EDM experiment will involve the detection of photons, Plexiglas $\mathrm{C}_{5} \mathrm{H}_{8} \mathrm{O}_{2}$ walls may provide a useful medium for getting the light to photomultiplier tubes. Additionally, Plexiglas would be an insulator for supporting the high voltage. Plexiglas does not provide the optimum neutron density, but it is reasonably good and has the additional advantage of saturating at a 2-cm wall thickness, a useful property since the entire source will need to be cooled to $0.5^{\circ} \mathrm{K}$ for the EDM experiment. As the Plexiglas will be cold, losses due to upscattering of cold neutrons by the hydrogen will be suppressed.

We found that the enhancement factor does not depend critically on the guide size, and we assumed for all calculations a square cross section of $10 \mathrm{~cm}$ on a side because that size is intended for the experiment at the Los Alamos National Laboratory.

\section{Results}

The engineering constraints imposed by the EDM experiment limit the vertical size of the source to $10 \mathrm{~cm}$ because of systematic errors due to UCN density gradients induced by the gravitation field of the Earth and because of limitations in the maximum high 
voltage that we can apply conveniently. The other two dimensions are constrained to be less than $20 \mathrm{~cm}$ for a cryostat of reasonable volume.

Rectangular and cylindrical geometries are compared in Fig. 2. When the neutrons are scattered into $4 \pi$, most of the solid angle is close to the wall. Thus most neutrons emerge from the material at a small angle to the surface. In the cylindrical geometry, this results in the neutrons traveling only a small distance before they intersect the wall again. In the rectangular geometry, this effect is reduced since the neutrons only intersect the next wall that is at right angles to the one from which they emerged. Hence, the rectangular geometry performs somewhat better than the cylindrical one. Both theoretical lines are above the simulated values but are well within the 40 percent accuracy of the theory.

Figure 3 shows an analogous plot using $\mathrm{C}_{5} \mathrm{H}_{8} \mathrm{O}_{2}$ (Plexiglas) as the wall material. Again, the agreement with the simulation is well within the 40 percent accuracy of the theory. The enhancement factors for $\mathrm{C}_{5} \mathrm{H}_{8} \mathrm{O}_{2}$ are smaller than those for $\mathrm{BeO}$. Even though the average diffusion length $l_{0}=\left[1 /\left(\sigma_{\text {inc }}+\sigma_{\mathrm{abs}}\right)\right]$ is smaller for Plexiglas, its absorption cross section is much larger than $\mathrm{BeO}$. Thus the neutrons are more likely to be absorbed in Plexiglas than in $\mathrm{BeO}$, the reflectivity is reduced, and the enhancement factor is smaller.

Finally, to determine the optimal dimensions of the rectangular geometry, we calculate the number of neutrons $M_{\mathrm{MC}}$, equal to the density times the volume, in the source as a function of the enhancement factor $E_{\mathrm{MC}}$ as shown in Fig. 4. The simulation demonstrates that it is best to maximize the vertical dimension; so it is fixed at $10 \mathrm{~cm}$. Figure 4 shows that the other two dimensions should be as large as practical because those 
values maximize the number of neutrons in the sample, the best condition for the EDM experiment.

\section{Conclusion}

Our Monte-Carlo simulation has shown that the density of a guide fed ${ }^{4} \mathrm{He}$ source can be increased by over a factor of two by surrounding the source with a reflector. In this simulation, we have tried to determine the best geometry and materials to maximize the gain. We have found that within the constraints of the EDM experiment, an enhancement of 2.2 seems to be realistic with a $10-\mathrm{cm} \times 10-\mathrm{cm}$ guide and with a cavity constructed from 2-cm thick Plexiglas with interior dimensions of $10 \mathrm{~cm}$ in height, $20 \mathrm{~cm}$ in width and $20 \mathrm{~cm}$ in length.

\section{References}

[1] R. Golub and J. M. Pendlebury, Phys. Lett. A 62 (1977) 337.

[2] R. Golub, D. S. Richardson, and S. K. Lamoreaux, Ultracold Neutrons (Adam Hilger Ltd., Bristol, 1991).

[3] R. Golub and S. K. Lamoreaux, Phys. Rep. 237 (1994) 1.

[4] J. Doyle and S. K. Lamoreaux, Europhys. Lett. 26 (1994) 253.

[5] Y. Ch. Yu, S. S. Malik, and R. Golub, Z. Phys. B 62 (1986) 137.

[6] J. M. Pendlebury, Kinetic Theory (Adam Hilger Ltd., Bristol, 1985).

[7] E. Fermi, A Course in Neutron Physics in Collected Papers, Volume II (The University of Chicago Press, Chicago, 1965). 
[8] S. F. Mughabghab, M. Divadeenam, and N. E. Holden, Neutron Cross Sections (Academic Press, New York, 1981). 


\section{Figure Captions}

Figure 1. The layout of the cold-neutron guide, the ${ }^{4} \mathrm{He}$ vessel, and the reflector.

Figure 2. The enhancement factor $E_{\mathrm{MC}}$ as a function of the source-size parameter $d$. For the rectangular geometry, $d$ is the length of the two horizontal dimensions, and for the cylindrical geometry, the diameter. The height is fixed at $10 \mathrm{~cm}$ and the guide is square with a $10-\mathrm{cm}$ side. The material is $\mathrm{BeO}$ of $5 \mathrm{~cm}$-thickness. The solid (dashed) lines are the theoretical prediction for rectangular (cylindrical) geometry.

Figure 3. The analogous calculation to Fig. 2 for 2-cm thick Plexiglas $\mathrm{C}_{5} \mathrm{H}_{8} \mathrm{O}_{2}$. The solid (dashed) curve belongs to the theory for rectangular (cylindrical) geometry.

Figure 4. The number of neutrons $M_{\mathrm{MC}}$ in the source is plotted as a function of the enhancement factor $E_{\mathrm{MC}}$. The points correspond to a rectangular geometry for a source with a height of $10 \mathrm{~cm}$, a guide with a sides of $10 \mathrm{~cm}$, and walls made of $2-\mathrm{cm}$ thick Plexiglas. The horizontal dimensions range from $20 \mathrm{~cm}$ to $10 \mathrm{~cm}$ in $1-\mathrm{cm}$ steps. 
Table 1

Material properties used in the calculations.

\begin{tabular}{cccc}
\hline Property & $\mathrm{BeO}$ & $\mathrm{H}_{2} \mathrm{O}$ & $\mathrm{C}_{5} \mathrm{H}_{8} \mathrm{O}_{2}$ \\
\hline$\sigma_{\text {abs }}$ (barns) & $40 \times 10^{-3}$ & 3.475 & 13.9 \\
$\sigma_{\text {inc }}$ (barns) & 20.0 & 164.0 & 698.0 \\
$\rho$ (No./cc) & $7.22 \times 10^{22}$ & $6.69 \times 10^{22}$ & $6.58 \times 10^{21}$ \\
\hline
\end{tabular}


Table 2

Dependence of enhancement factor on thickness.

\begin{tabular}{|c|c|c|c|}
\hline Thickness (cm) & $\mathrm{BeO} E_{\mathrm{MC}}$ & $\mathrm{H}_{2} \mathrm{O} E_{\mathrm{MC}}$ & $\mathrm{C}_{5} \mathrm{H}_{8} \mathrm{O}_{2} E_{\mathrm{MC}}$ \\
\hline 1 & 1.424 & 1.980 & 1.854 \\
\hline 2 & 1.795 & 1.984 & 2.021 \\
\hline 3 & 2.113 & 1.984 & 0.033 \\
\hline 4 & 2.369 & 1.984 & 2.034 \\
\hline 5 & 2.518 & 1.984 & 2.039 \\
\hline 6 & 2.710 & 1.984 & 2.039 \\
\hline 7 & 2.811 & 1.984 & 2.039 \\
\hline 8 & 2.888 & 1.984 & 2.039 \\
\hline 9 & 2.941 & 1.984 & 2.039 \\
\hline sat. thickness & $6 \mathrm{~cm}$ & $2 \mathrm{~cm}$ & $2 . \mathrm{cm}$ \\
\hline$\beta$ & 0.863 & 0.912 & 0.906 \\
\hline$E_{t}$ & 2.810 & 2.286 & 2.277 \\
\hline difference $(\%)$ & 3.6 & 13.2 & 11.0 \\
\hline
\end{tabular}




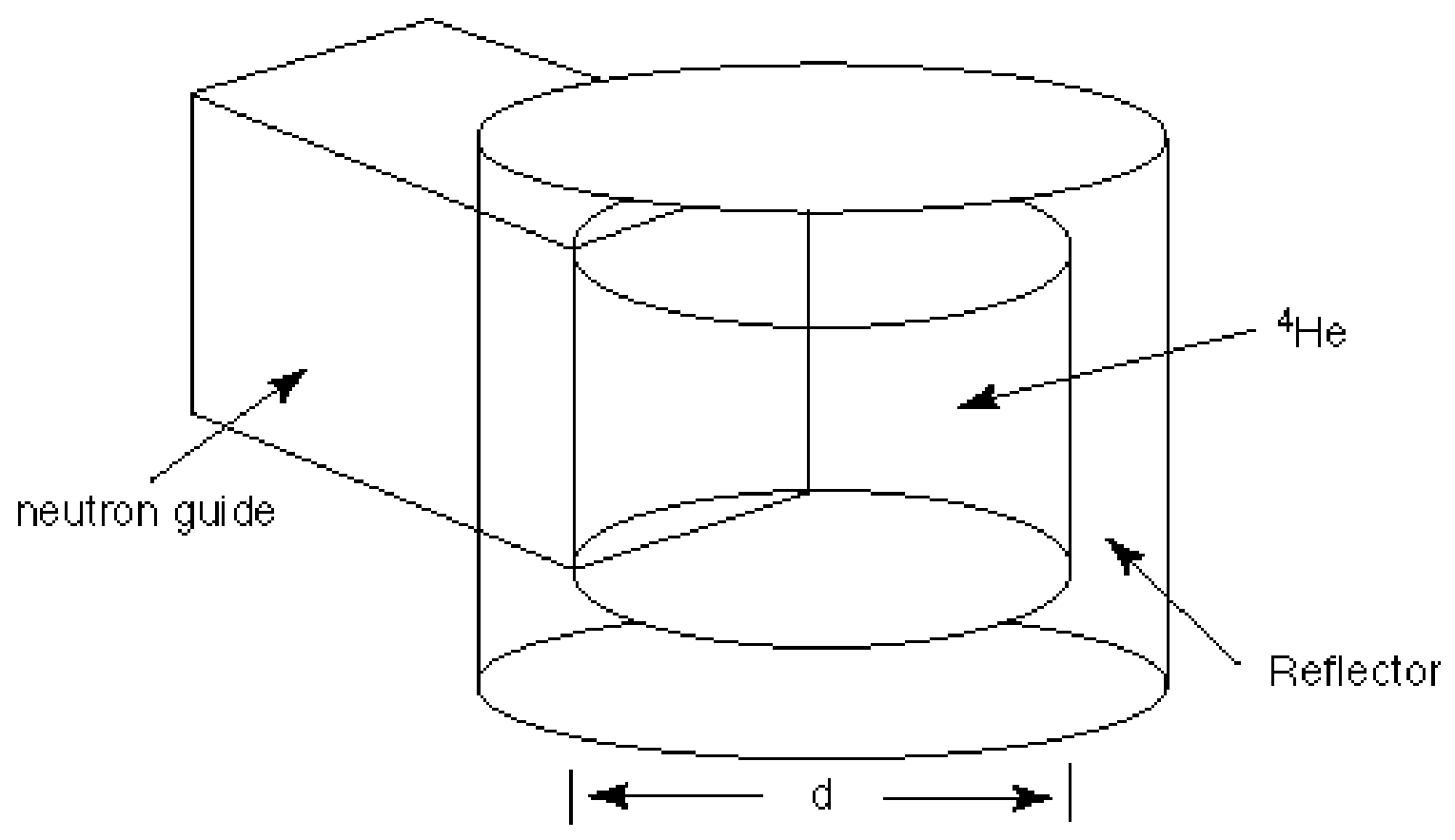

Fig. 1 


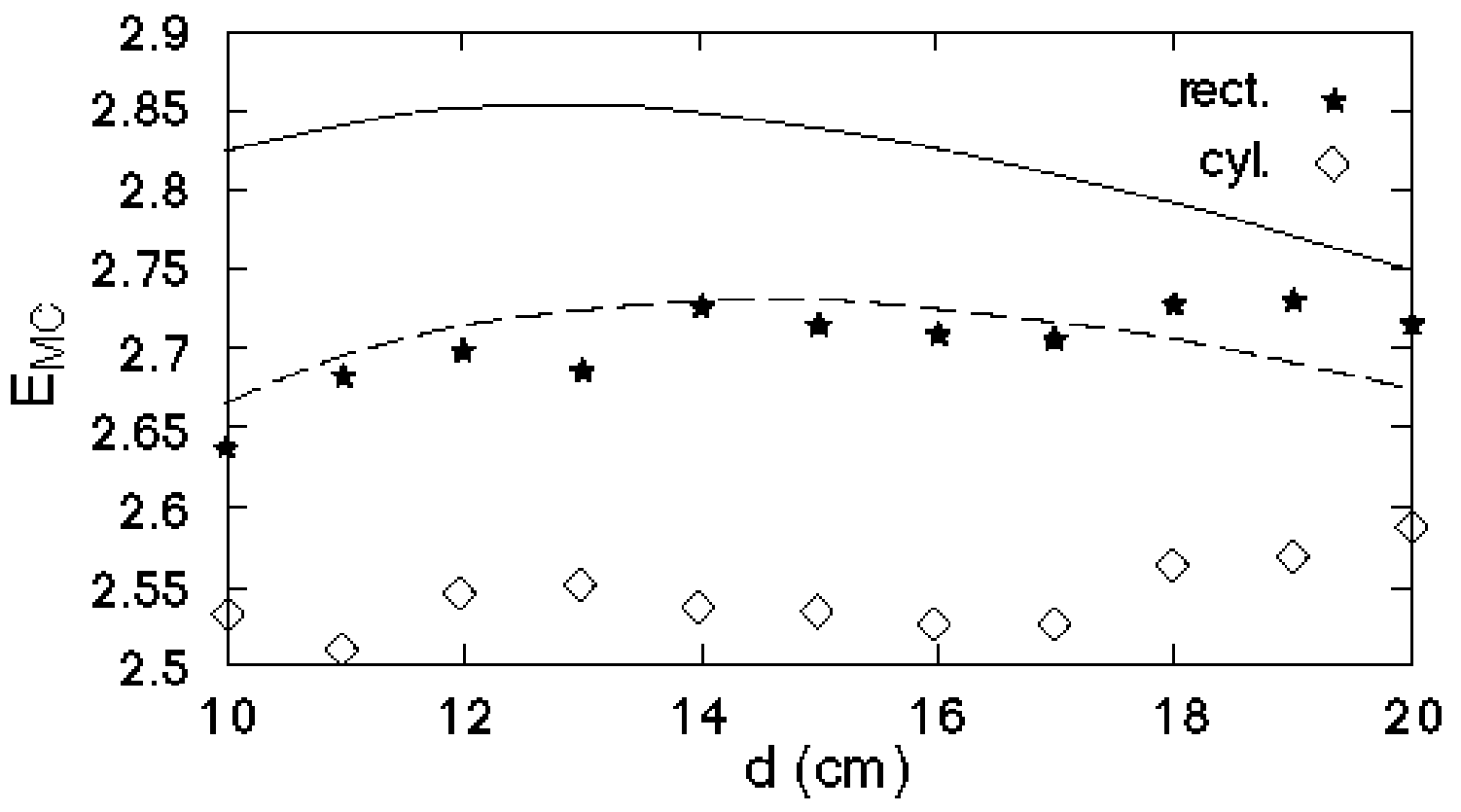

Fig. 2 


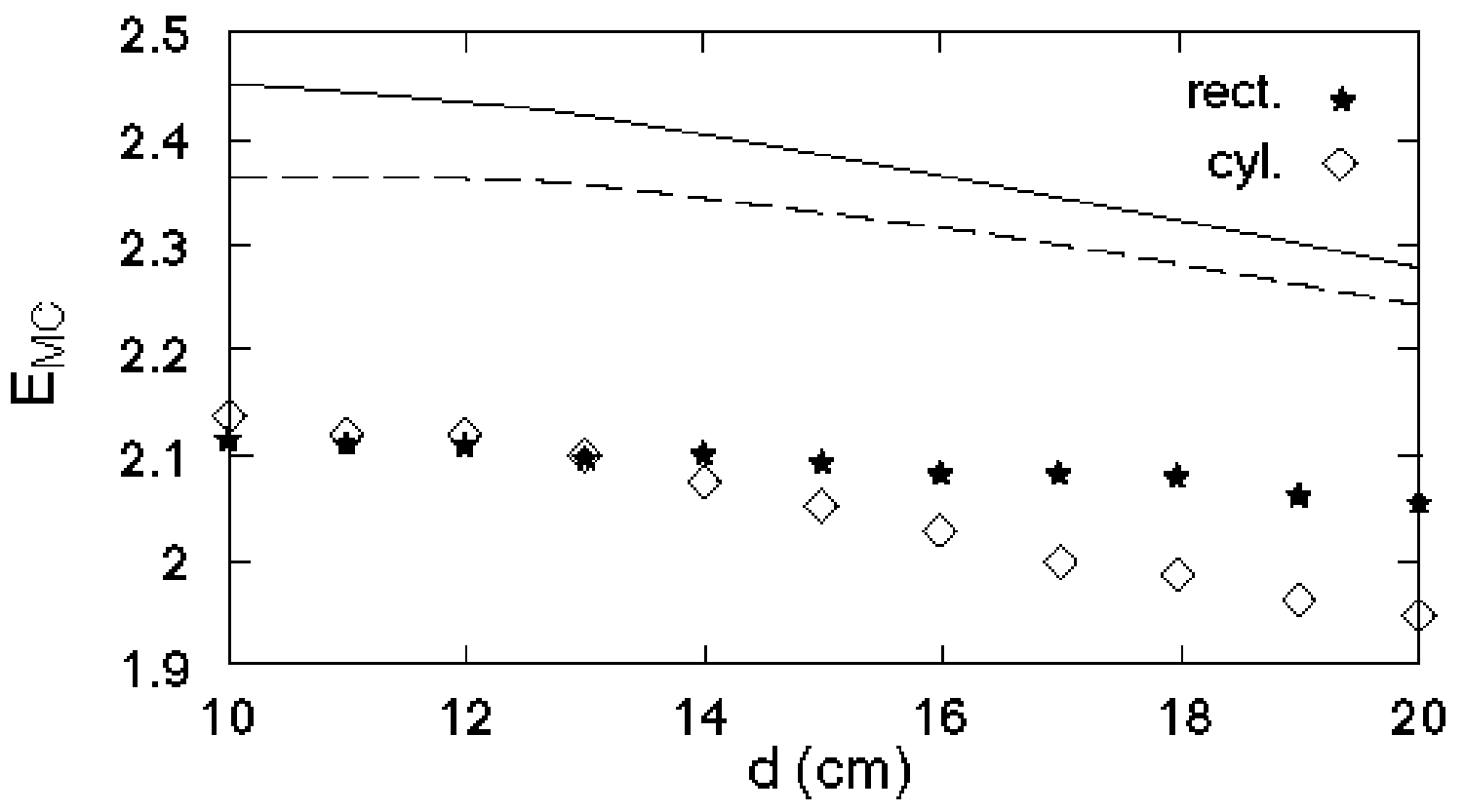

Fig. 3 


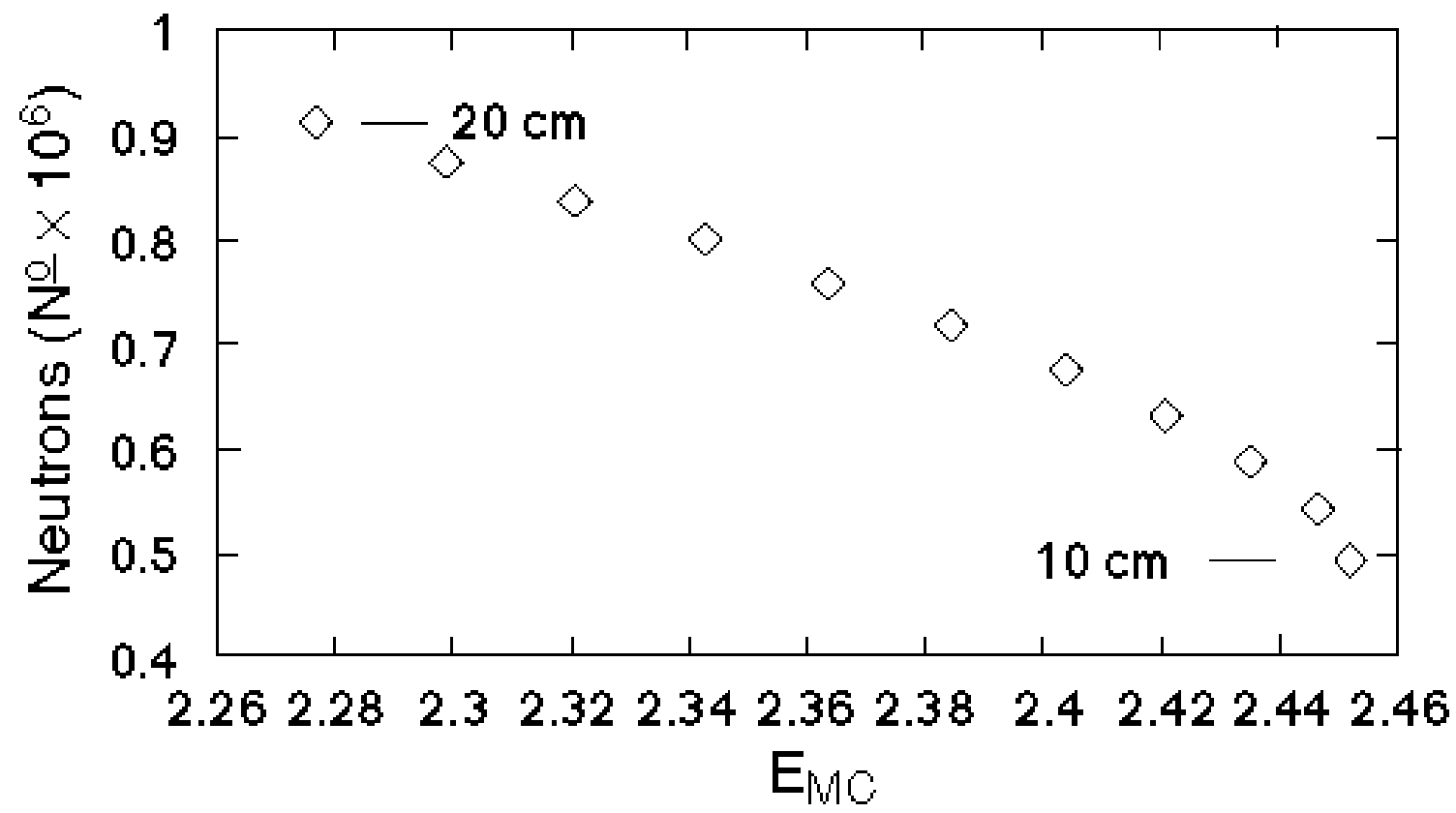

Fig. 4 